\title{
News media coverage of COVID-19 public health and policy information
}

Katharine J. Mach ${ }^{1,2 凶}$, Raúl Salas Reyes (10 ${ }^{3}$, Brian Pentz (10 ${ }^{3}$, Jennifer Taylor (10 ${ }^{4}$, Clarissa A. Costa ${ }^{3}$, Sandip G. Cruz ${ }^{3}$, Kerronia E. Thomas ${ }^{3}$, James C. Arnott (10 ${ }^{5}$, Rosalind Donald ${ }^{1}$, Kripa Jagannathan (1) ${ }^{6,7}$, Christine J. Kirchhoff ${ }^{8}{ }^{8}$, Laura C. Rosella (i) ${ }^{9}$ \& Nicole Klenk (i) ${ }^{3}$

During a pandemic, news media play a crucial role in communicating public health and policy information. Traditional newspaper coverage is important amidst increasing disinformation, yet uncertainties make covering health risks and efforts to limit transmission difficult. This study assesses print and online newspaper coverage of the coronavirus disease COVID-19 for March 2020, when the global pandemic was declared, through August 2020 in three countries: Canada (with the lowest per-capita case and death rates during the study timeframe), the United Kingdom (with a pronounced early spike), and the United States (with persistently high rates). Tools previously validated for pandemic-related news records allow measurement of multiple indicators of scientific quality (i.e., reporting that reflects the state of scientific knowledge) and of sensationalism (i.e., strategies rendering news as more extraordinary than it really is). COVID-19 reporting had moderate scientific quality and low sensationalism across 1331 sampled articles in twelve newspapers spanning the political spectrums of the three countries. Newspapers oriented towards the populist-right had the lowest scientific quality in reporting, combined with very low sensationalism in some cases. Against a backdrop of world-leading disease rates, U.S. newspapers on the political left had more exposing coverage, e.g., focused on policy failures or misinformation, and more warning coverage, e.g., focused on the risks of the disease, compared to U.S. newspapers on the political right. Despite the generally assumed benefits of low sensationalism, pandemicrelated coverage with low scientific quality that also failed to alert readers to public-health risks, misinformation, or policy failures may have exacerbated the public-health effects of the disease. Such complexities will likely remain central for both pandemic news media reporting and public-health strategies reliant upon it.

\footnotetext{
${ }^{1}$ Department of Environmental Science and Policy, Rosenstiel School of Marine and Atmospheric Science, University of Miami, Miami, FL, USA. ${ }^{2}$ Leonard and Jayne Abess Center for Ecosystem Science and Policy, University of Miami, Coral Gables, FL, USA. ${ }^{3}$ Department of Physical and Environmental Sciences, University of Toronto, Toronto, ON, Canada. ${ }^{4}$ Department of Geography and Planning, University of Toronto, Toronto, ON, Canada. ${ }^{5}$ Aspen Global Change Institute, Basalt, CO, USA. ${ }^{6}$ Earth and Environmental Sciences Area, Lawrence Berkeley National Laboratory, Berkeley, CA, USA. ${ }^{7}$ School for Environment and Sustainability, University of Michigan, Ann Arbor, MI, USA. ${ }^{8}$ Department of Civil and Environmental Engineering, University of Connecticut, Storrs, CT, USA. ${ }^{9}$ Dalla Lana School of Public Health, University of Toronto, Toronto, ON, Canada. ${ }^{凶}$ email: kmach@miami.edu
} 


\section{Introduction}

ews media reporting is understood to play a central role during national security and health emergencies (Laing, 2011; Klemm et al., 2016; Pieri, 2019). News coverage communicates risks to readers and shapes public perceptions through the amount, content, and tone of reporting. It simultaneously frames ongoing public debates about policy responses, including conflicting priorities relevant to the timing or stringency of implemented policies (Laing, 2011; Pieri, 2019). Pandemic policy-making requires rapid, iterative responses under conditions of knowledge deficit, as well as the coordination of multi-level public-health agencies and sectors (e.g., hospitals, schools, and workplaces) (Laing, 2011; Rosella et al., 2013). In these complex circumstances, news media serve as a primary source of health information and uncertainties and connect health professionals, policymakers, and the public in critical ways (Laing, 2011; Hoffman and Justicz, 2016). The quality and balance of scientific coverage, such as through reporting that reflects the state of scientific knowledge and is not overstated, affect trust in science and accountability for decision-making (Laing, 2011; Klemm et al., 2016; Hoffman and Justicz, 2016).

Inadequate scientific quality in news coverage of past pandemics has posed risks and limited capacities to disseminate public-health guidance and coordinate responses (Hoffman and Justicz, 2016). Reporting on the state of scientific knowledge during a novel, evolving pandemic is challenging. Low-quality scientific reporting of pandemics may overstate or understate disease risks or the efficacy of protective measures for different individuals or fail to communicate the nature of the evidence. Such reporting may constrain the feasibility or effectiveness of options for policymakers directing government action, miss opportunities to inform individuals making health decisions, and increase the exposure of health professionals to disease. It can both exacerbate disease outcomes and generate unnecessary fear, in combination with other factors shaping perceptions among the public (Laing, 2011; Klemm et al., 2016; Hoffman and Justicz, 2016). For example, news media reporting may have overly emphasized the threat of the 2009 A/H1N1 influenza (H1N1) pandemic with insufficient indication of available protective measures, and in pairing trustworthy information from credible scientists with uninformed opinions, it may have promoted a "false balance" (Laing, 2011; Klemm et al., 2016; Hoffman and Justicz, 2016). Further, news coverage rapidly waned after the initial pandemic declaration even though public-health risks persisted (Klemm et al., 2016; Reintjes et al., 2016). Similar issues with media reporting occurred during the 2003 severe acute respiratory syndrome (SARS) outbreak and the 2014 Ebola outbreak (Hoffman and Justicz, 2016; Pieri, 2019).

During the COVID-19 pandemic, media representations of complex, rapidly evolving epidemiological science shape public understandings of the risks, measures to limit disease spread, and associated political and policy discourses. Traditional newspaper media coverage may have particular importance given simultaneous misinformation and disinformation, social fragmentation, political polarization, and failures of policy coordination, and national newspapers influence how other outlets cover the same subject across media platforms (Ball and Maxmen, 2020; Holtz et al., 2020; Thorp, 2020; Grossman et al., 2020). The COVID-19 pandemic creates an opportunity to assess the strengths and limitations of the media's pandemic coverage and provide insights for future news media coverage. Such assessment also informs the communication strategies of public-health institutions and policymakers towards clear public-health guidance and coordinated responses across health systems (Laing, 2011; Hoffman and Justicz, 2016; Pieri, 2019).

Canada, the United Kingdom, and the United States, our countries of focus, differ in how they govern public health, including pandemic responses. In its constitutionally determined role, the Canadian federal government sets healthcare standards and administers funding to support the healthcare system spanning provinces and territories (Government of Canada, 2016). Pandemic health-related policies are set and implemented predominantly by provinces with federal guidance from Health Canada and the Public Health Agency of Canada (Adeel et al., 2020). The U.K. central government funds healthcare throughout the United Kingdom yet only sets policies for England. Northern Ireland, Scotland, and Wales each govern their own National Health Service systems. By contrast, the healthcare system in the United States is a complex mixture of public and private health insurance programs. The U.S. federal government generally adopts a leading role during national crises, although during the COVID-19 pandemic states and municipalities have led adoption and implementation of most policy measures to contain the spread of COVID-19 (Adeel et al., 2020). Prior to the COVID-19 pandemic, the 2019 Global Health Security Index ranked the United States first, United Kingdom second, and Canada fifth among 195 countries for preparedness to manage a serious disease outbreak (Cameron et al., 2019).

In this paper, we systematically quantify the amount, scientific quality, and sensationalism of newspaper media coverage of COVID-19 in Canada, the United Kingdom, and the United States. Newspapers studied span the political spectrum of each case-study country (Table 1) (Gentzkow and Shapiro, 2010; Puglisi and Snyder, 2015; Anderson and Coletto, 2017; Mitchell et al., 2018; Hönnige et al., 2020; Jurkowitz et al., 2020; Austen, 2020). Our analysis begins two weeks prior to COVID-19's official recognition as a pandemic and follows its development over the subsequent five months (i.e., from 1 March 2020 to 15 August 2020). Given the volume of COVID-19 news media articles published over the timeframe of this study, we created a manageable corpus for analysis by randomly sampling one day of media coverage per week for six consecutive 4-week periods; we then randomly selected five eligible articles from each news outlet on each sampled day for the evaluation of scientific quality and sensationalism. In our evaluation, scientific quality refers to the alignment between reporting and the state of scientific evidence and its uncertainties, and sensationalism is a discursive strategy

Table 1 News media outlets studied, by country of publication and political orientation.

\section{Country of publication Political orientation of newspaper}

Left $\leftrightarrow$ Right

Canada

United Kingdom

Toronto Star

United States

The Guardian

The New York Times

The Globe and Mail
The Times of London
The Washington Post

The National Post

The Telegraph

The Wall Street Journal
Toronto Sun

Daily Mail

New York Post

Based on media slant and readership, news outlets are ordered across the political spectrum of each country. Note that relative placements are specific to each country context. 
rendering news as more extraordinary, interesting, or relevant than it really is (Oxman et al., 1993; Molek-Kozakowska, 2013; Hoffman and Justicz, 2016). We apply previously validated survey tools developed to measure scientific quality and sensationalism of pandemic-related health news records in combination with broader methods from policy analyses of pandemic responses (SI Coding Tool) (Oxman et al., 1993; Rosella et al., 2013; MolekKozakowska, 2013; Reintjes et al., 2016; Hoffman and Justicz, 2016). We analyze (1) the COVID-19 public-health outcomes and policies in each country and (2) the amount, scientific quality, sensationalism, and topics of COVID-19 news media coverage across the political spectrum of each country.

\section{Methods}

Public health contextualization of news media analyses. To contextualize our news media analyses, we analyzed and visualized existing data sets on the number of COVID-19 cases, deaths, and tests in each country (e.g., Roser et al., 2020; CBC News, 2020; Public Health England and NHSX, 2020; CDC, 2020). We also recorded the key public-health declarations, policies, and guidance during the study time period (e.g., drawing from WHO, 2020a, 2020b; see also SI Table S1). We tracked these decisions at international scales through to subnational scales in each country studied. Media analyses outlined below thereby were considered with respect to the reported number of cases and confirmed deaths and policy actions taken (Reintjes et al., 2016).

News media search strategy and inclusion criteria. Print and online news media records were retrieved from the Factiva database for news outlets across the political spectrum of Canada, the United Kingdom, and the United States (see Table 1) (Gentzkow and Shapiro, 2010; Puglisi and Snyder, 2015; Anderson and Coletto, 2017; Mitchell et al., 2018; Hönnige et al., 2020; Jurkowitz et al., 2020; Austen, 2020). Selected news media outlets have primary news products in print and online media, rather than television broadcasting or social media, and full article entries available in Factiva. Search terms included "coronavirus," "COVID-19," “epidemic," "outbreak," "pandemic," or "SARSCoV-2." Individual English-language news articles were retrieved for sampled dates between 1 March 2020 and 15 August 2020. This period captures news media coverage prior to the declaration of the COVID-19 pandemic and over the subsequent five months.

Individual news records were screened to identify original news reporting (i.e., news reporting and news analysis articles) relevant to our study objectives. First, eligible articles must have a direct focus on the public-health implications of COVID-19 or on attempts to control its spread-in some or all of an article's text. By excluding articles without this focus, we ensured all articles included in the study could contain scientific information on the public health effects or spread of COVID-19 and associated policies. Second, eligible articles must be focused on the newspaper's country of publication (e.g., an article reporting on COVID-19 transmission or mitigation efforts in only New Zealand or China, without discussion of implications for the newspaper's country of publication, would be excluded). We included this eligibility criterion to analyze science-policy interfaces and science-society interactions most proximate to the news outlets, although we acknowledge that articles about other countries may influence perceptions of readers even without direct discussion of implications for them. Third, eligible articles must be original news reporting or analysis, meaning we excluded opinion pieces, editorials, interview transcripts, microblogs, front-page snippets, news roundups, obituaries, advertisements, corrections memos, and letters to the editor; these excluded article types would have required distinct question framings beyond the scope of our codebook. This third criterion, therefore, ensured that coded responses could be compared coherently across articles for the different measures of scientific quality and sensationalism.

Sampling of news media articles. As the evaluation of scientific quality and sensationalism through manual coding is time intensive, and a very large number of COVID-19 news media articles were published during the timeframe of our study, we used a random sample of news media articles for analysis, prioritizing sampling during each week over the course of the study timeframe. The sample design enabled a manageable analysis of newspaper media coverage and potential changes over the timeframe of the study. First, the sample of news media articles was constructed by sampling one day of media coverage per week in consecutive four-week periods. These four days of the week were randomly sampled without replacement (Monday through Saturday only, not including Sunday in the sampling), given cyclic variation in news media coverage (Lacy et al., 2001; Riffe et al., 2016). The study timeframe was divided into six four-week periods of equal duration from 1 March to 15 August 2020.

Second, for each randomly sampled day, all available news records were retrieved from Factiva for the 12 news outlets (Table 1). Randomly selected articles were screened for eligibility, with the goal of identifying 5 eligible articles for each news outlet on each sampled day. In some cases, fewer than 5 eligible articles were published by a given outlet on a sampled day. In these cases, the full set of eligible articles was included in the study.

Analysis of scientific quality and sensationalism of news articles. The coding tool for measuring scientific quality and sensationalism of news article records was adapted from the final tool of Hoffman and Justicz, designed for evaluating pandemic-related health news records (Hoffman and Justicz, 2016). Scientific quality, as defined in that study, is "a measure of an article's reliability and credibility on a given topic" (Hoffman and Justicz, 2016). Importantly, scientific quality is linked to the state of scientific understanding and its uncertainties at specific moments in time rather than being an absolute or objective characteristic. The codebook we applied for measuring scientific quality is therefore designed to be flexible and responsive to the inevitable shifts in scientific understanding that occur through time, most especially during a novel disease outbreak and evolving pandemic. Sensationalism, as defined in that study, is "a way of presenting articles to make them seem more interesting or extraordinary than they actually are" (Hoffman and Justicz, 2016).

Our coding tool (SI Coding Tool) included six questions for scientific quality (each evaluated on a scale from 1 to $5-5$ corresponding to highest quality) and six questions for sensationalism (each evaluated on a scale from 1 to $5-5$ corresponding to highest sensationalism). The question categories (SI Coding Tool) for assessing scientific quality were as follows: applicability, opinion versus facts, validity, precision, context, and global assessment (i.e., an overall assessment of the article's scientific quality based on the five preceding specific measures). For sensationalism, the question categories (SI Coding Tool) included exposing, speculating, generalizing, warning, extolling, and global assessment (i.e., an overall assessment of the degree of sensationalism in the article based on the five preceding specific measures) (Oxman et al., 1993; Molek-Kozakowska, 2013; Hoffman and Justicz, 2016).

In addition, metadata collected for each article included the coder's identity, the article title, the article's sample date, the news 
outlet (including if the article was originally written by another outlet such as the Associated Press), the societal sector (up to 2 selected per article), and public-health measures discussed (SI Coding Tool).

Coder assignments, training, reliability assessment, and analysis. For each sampled day, two independent coders assessed all relevant news media records based on the scientific quality and sensationalism questions and article-attribute metadata. Coders recorded scores for each article through a Google-form version of the codebook (SI Coding Tool).

To ensure consistent application of the coding tool, substantial training and calibration occurred over a six-week period. First, the three coders in coordination with the project leadership team read national and international public-health agency descriptions of the coronavirus disease and associated public-health policies and measures. Second, the coders completed multiple rounds of individual coding of example news articles, followed by group discussions of application of the codebook. The group discussions considered difficult judgments and common versus unusual examples. The goal was to ensure consistent application of the coding tool across question categories and the range of article examples that arose. During the training and calibration phases of coding, we updated the codebook to include examples specific to news records on COVID-19 (SI Coding Tool), and we tracked illustrative examples (news articles and specific quotes) across the scale (1-3-5) for the scientific quality and sensationalism question categories. This process led to development of example answers particularly representative of low versus high scientific quality and low versus high sensationalism under each category of response. Additionally, we developed "decision rules" for the more unusual or challenging categories of examples to ensure consistency across coders, especially where disagreements arose in individually assigned responses.

Interrater reliability was assessed during the training and calibration stage and throughout the duration of the study. Where coders assigned scores for a given question that were 3 or 4 units apart on the 1-5 scale, a reconciliation discussion occurred; the small fraction of question responses in this category following the training stage enabled the coders and project team to continue developing and ensuring shared understanding of coding approaches for unusual or challenging applications. Weighted Cohen's Kappa, with quadratic weighting, was applied given the high-inference codebook and ordinal data collected via a Likert scale, as previously done for related measures (Cohen, 1960; Fleiss and Cohen, 1973; Oxman et al., 1993; Antoine et al., 2014; Hoffman and Justicz, 2016; Tran et al., 2020). Coded data were analyzed with Kruskal-Wallis one-way analysis of variance and post-hoc multi-comparison pairwise tests (kruskal.test and kruskalmc in pgirmess package in R) (Giraudoux et al., 2018; R Core Team, 2020).

\section{Results}

Public health and policy contexts. From March through August 2020, Canada, the United Kingdom, and the United States differed substantially in their public-health responses to COVID-19 and in health outcomes from the novel coronavirus disease (Fig. 1). Beginning in early March, all three countries implemented a combination of policy measures to contain the spread of COVID-19, including emergency laws, stay-at-home orders, mask mandates, school and business closures, border and travel restrictions, social distancing measures, and quarantines upon entry (SI Table S1). These restrictions were followed by gradual phases of reopening measures allowing restricted social and economic activities to occur. Across the

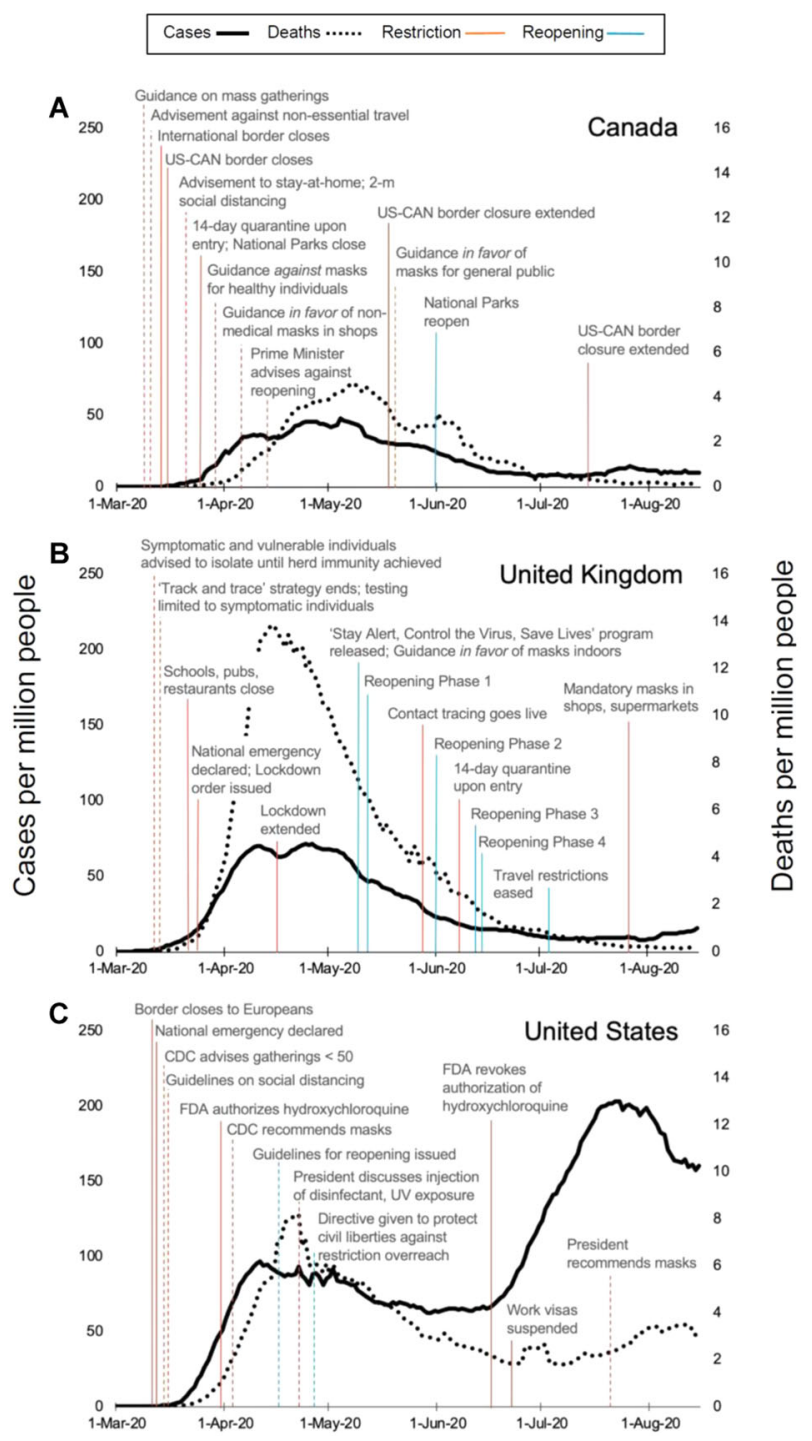

Fig. 1 COVID-19 cases, deaths, and national-level policies. COVID-19 cases, deaths, and national-level policies are indicated for (A) Canada, (B) the United Kingdom, and (C) the United States. 7-day rolling averages of cases (left vertical axis, solid black line) and deaths (right vertical axis, dotted black line) per one million people are shown for the timeframe of this media study, 1 March through 15 August 2020 (Roser et al., 2020). The timeline for each country specifies national-level public-health policies and guidance, especially emergency declarations, school and non-essential business closures, travel and border restrictions, quarantines and social distancing, mask usage, and reopening phases. Implementation of enforceable policies (solid) and non-enforced guidance (dotted) is specified with vertical red lines, and corresponding reopening and relaxation of policies and guidance are specified with vertical blue lines. Detailed descriptions of national-level policies within each panel are provided in SI Table S1.

three countries, the role of national versus subnational governments differed with respect to authority and actions on public-health guidance and care, resulting in differing timing and levels of coordination for both restrictions and reopening measures (SI Fig. S1). From March to August 2020, the United Kingdom experienced the highest death rate from COVID-19 (maximum 7-day average of 13.9 deaths per million people; Fig. 1), whereas the United States had the highest case rate of the three countries (maximum 7-day average of 203.5 cases per 


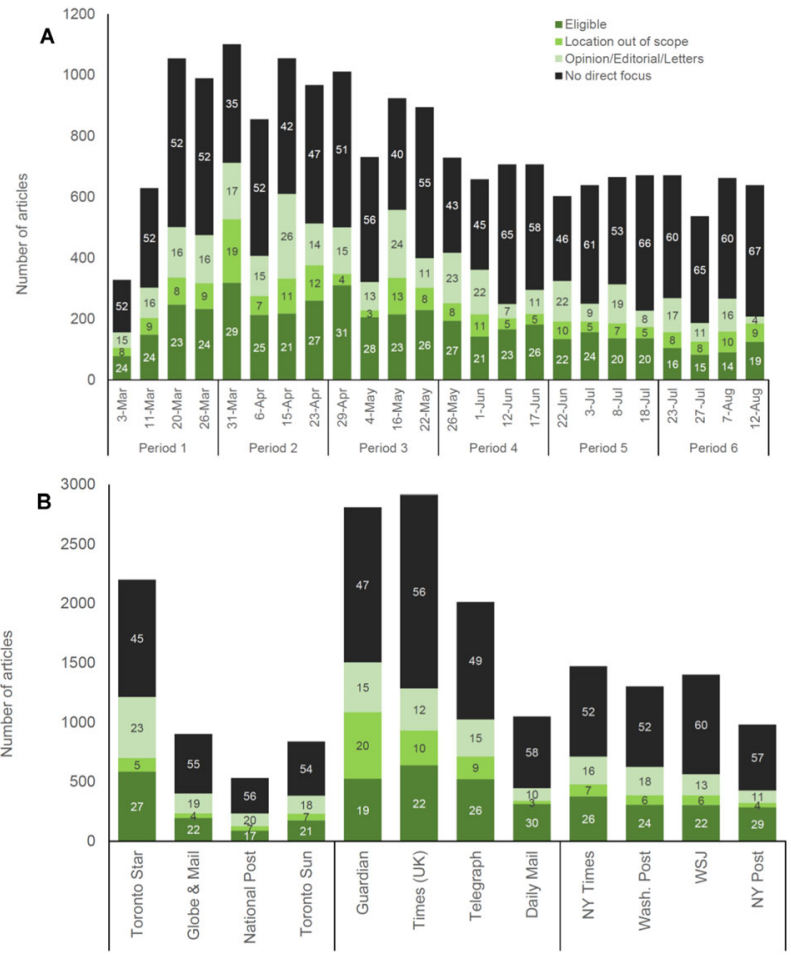

Fig. 2 COVID-19 news media articles published over the timeframe of this study and by news outlet. For each randomly sampled day (A) and each news outlet (B), the total number of individual news records is shown, based on Factiva database searches for articles related to COVID-19 public health and policy information (Methods). News articles are partitioned across the following categories: articles eligible for inclusion in our study (eligible), articles not focusing on the newspaper's country of publication (location out of scope), articles that are not original news reporting or analysis (opinion/editorial/letters), and articles that include COVID-19relevant search terms, but do not include any direct focus on COVID-19 public health or policy information (no direct focus). Estimated totals for these categories are calculated using (i) the total number of Factiva returns and (ii) the rates at which articles were assigned to these categories during the eligibility screening process for each outlet and randomly sampled day (SI Fig. S4). On the stacked bars, percentages of articles falling into each category are specified for each day (A) and news outlet (B).

million), as well as the greatest cumulative number of cases and deaths globally (SI Figs. S2-S3). Of the three countries, Canada had the most effective public-health outcomes as measured by per capita COVID-19 case or death rates (Fig. 1).

The amount of pandemic media coverage. The studied news outlets differed in the amount of news media coverage related to COVID-19 from 1 March through 15 August 2020 (Fig. 2). The amount of coverage increased notably in March as case rates climbed in each country, subsequently decreasing gradually in May and June while case rates also declined. Across the 24 randomly sampled days, the 12 studied news outlets published 18,430 articles related to COVID-19. Of these, an estimated 4321 articles (23.4\%) were eligible for inclusion in this study-that is, as news reporting or analysis relevant to the country of publication and containing a direct focus on COVID-19 public health or policy information (SI Figs. S4-S5). Articles with a direct focus on COVID-19 public health or policy information (to a small or large extent) could be coded for the scientific quality of the reporting of this information and its sensationalism.
Content analysis of pandemic media coverage. We collected a manageable, well-defined random sample of 1331 news media articles satisfying our eligibility criteria (SI Fig. S4) for coding of scientific quality and sensationalism (SI Coding Tool and Dataset S1). Six questions each for scientific quality and for sensationalism were evaluated on a scale from 1 to 5 ( 5 corresponding to highest scientific quality or sensationalism, 1 corresponding to lowest scientific quality or sensationalism). Question categories included for scientific quality: applicability, opinion versus facts, validity, precision, context, and global assessment (i.e., an overall assessment of the article's scientific quality); and for sensationalism: exposing, speculating, generalizing, warning, extolling, and global assessment (i.e., an overall assessment of the degree of sensationalism in the article) (SI Coding Tool). For this content analysis, interrater reliability was moderate to substantial for the summative "global" assessment of scientific quality and sensationalism (SI Table S2). Reliability was similarly high for specific scientific quality and sensationalism measures, with the exception of questions for which coded scores displayed restriction of range or unbalanced distributions (e.g., "generalizing" scores of mostly 1 and 2, rather than ranging from 1 through 5 with balance around 3; SI Coding Tool and Dataset S1) (Hallgren, 2012; Tran et al., 2020).

The scientific quality of pandemic media coverage. The scientific quality of news media articles differed among news outlets across the political spectrums of the respective countries (Fig. 3). Within each country, the overall scientific quality of news reporting and analysis was lowest on the populist-right of the political spectrum (mean summative "global" scientific quality of $2.58, \mathrm{n}=106$ articles, for Toronto Sun; $2.67, \mathrm{n}=115$, for Daily Mail; and 2.28, $\mathrm{n}=118$, for New York Post; $p \leq 0.001$ for Kruskal-Wallis, $p \leq 0.05$ for within-country pairwise comparisons except Daily Mail versus Times of London and Telegraph, SI Table S3). For these outlets, lower scientific quality was especially evident for validity, precision, and context as measures of scientific quality (e.g., articles reporting claims without fact checking, specificity, or background details) (Fig. 3).

The sensationalism of pandemic media coverage. The sensationalism of news media articles was low overall for all news outlets, although somewhat greater for outlets on the left and middle of the political spectrum in Canada and the United States (Fig. 4F). In both countries, news outlets at the populist-right combined low scientific quality with low sensationalism (Figs. 3F and $4 \mathrm{~F}$ ). In Canada, the overall sensationalism of news reporting and analysis was lowest for the Toronto Sun (mean summative "global" sensationalism of 1.77, $n=106$ articles; $p \leq 0.001$ for Kruskal-Wallis, $p \leq 0.05$ for pairwise comparisons with Globe and Mail and National Post, SI Table S3). In the United States, overall sensationalism was lower in the Wall Street Journal (mean global sensationalism of 2.03, $n=118$ articles) and New York Post (mean of 2.13, $n=118$ ), as compared to the New York Times (mean of 2.40, $n=120$ ) and Washington Post (mean of 2.38, $n=119 ; p \leq 0.001$ for Kruskal-Wallis, $p \leq 0.05$ for pairwise comparisons, SI Table S3). For these outlets, lower sensationalism was especially observed for exposing, speculating, and warning as measures of sensationalism (Fig. 4). In the United Kingdom, overall sensationalism did not vary across news outlets $(p=0.283$ for Kruskal-Wallis, SI Table S3).

Syndicated versus original reporting. Across all outlets, the scientific quality of original reporting (mean global scientific quality of $2.93, n=1278$ articles) was significantly higher than the scientific quality of syndicated articles (mean of 2.71, n=54; 

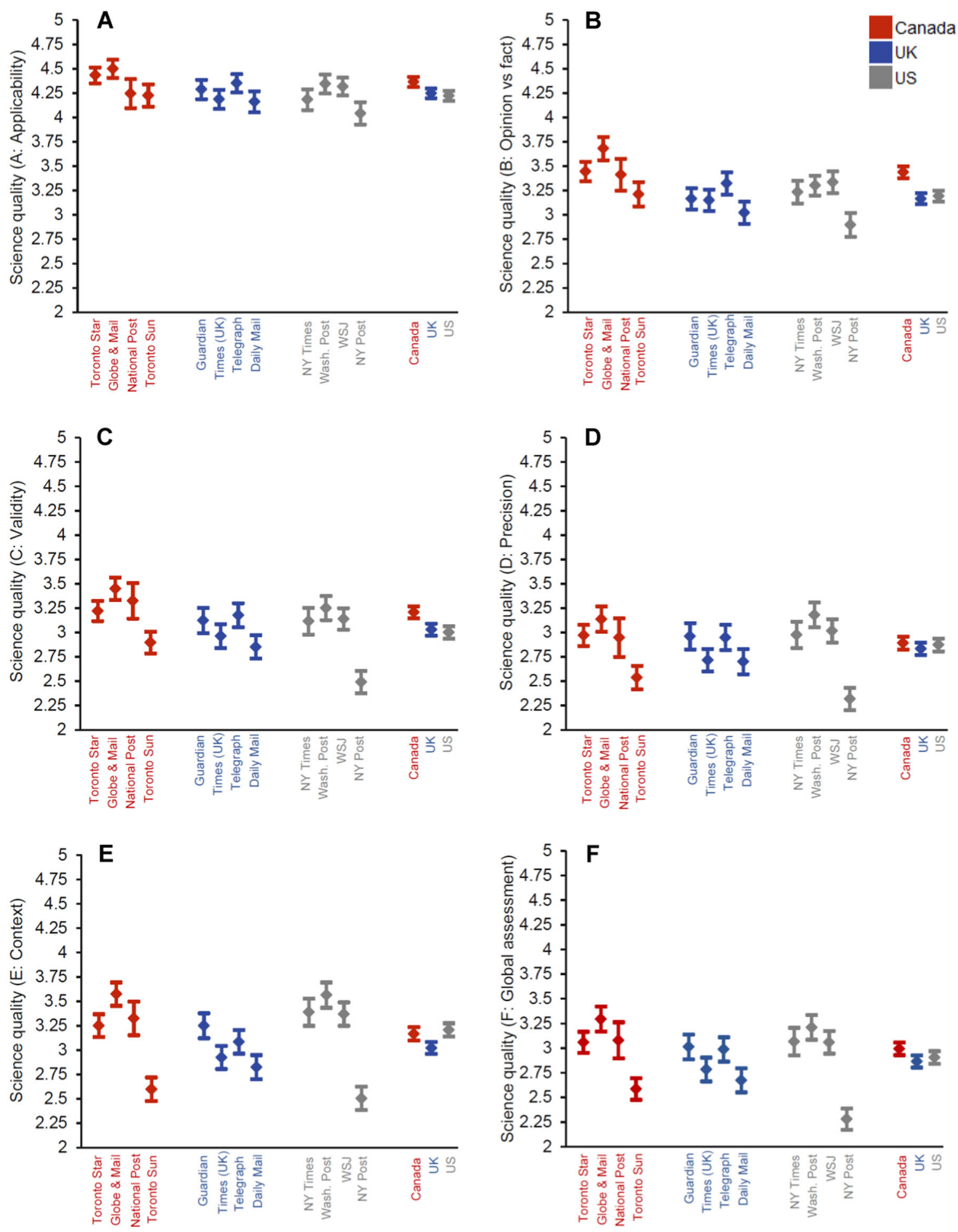

Fig. 3 Scientific quality of news media articles by news outlet and country of publication. Scores for six scientific quality questions (SI Coding Tool) are shown (mean, 95\% confidence interval) for articles $(n=1331)$ communicating COVID-19 public health or policy information (Fig. 2): (A) applicability, (B) opinion versus facts, $(\mathbf{C})$ validity, (D) precision, (E) context, and (F) global assessment (i.e., an overall assessment of the article's scientific quality). Each question was evaluated on a scale from 1 to 5 (5 corresponding to highest scientific quality). Sampled articles were published between 1 March and 15 August 2020 (Methods). Kruskal-Wallis one-way analysis of variance and post-hoc multi-comparison test statistics are in SI Table S3.

$p=0.020$, Kruskal-Wallis; SI Fig. S6 and Table S4). Additionally, the sensationalism of syndicated articles (mean global sensationalism of $1.82, n=54$ articles) was significantly lower than the sensationalism of original reporting (mean of 2.14, $n=1278$; $p \leq 0.001$, Kruskal-Wallis; SI Fig. S6 and Table S4). The Toronto Sun published the highest proportion of syndicated news articles by far, with $34 \%$ of the paper's 106 coded articles originating from syndicated sources. Other news outlets with more than $1 \%$ of coded articles drawing from syndicated sources included the Toronto Star (6\% of articles) and the National Post (11\%).

Neither scientific quality nor sensationalism varied substantially through time, with the exception of lower scientific quality 

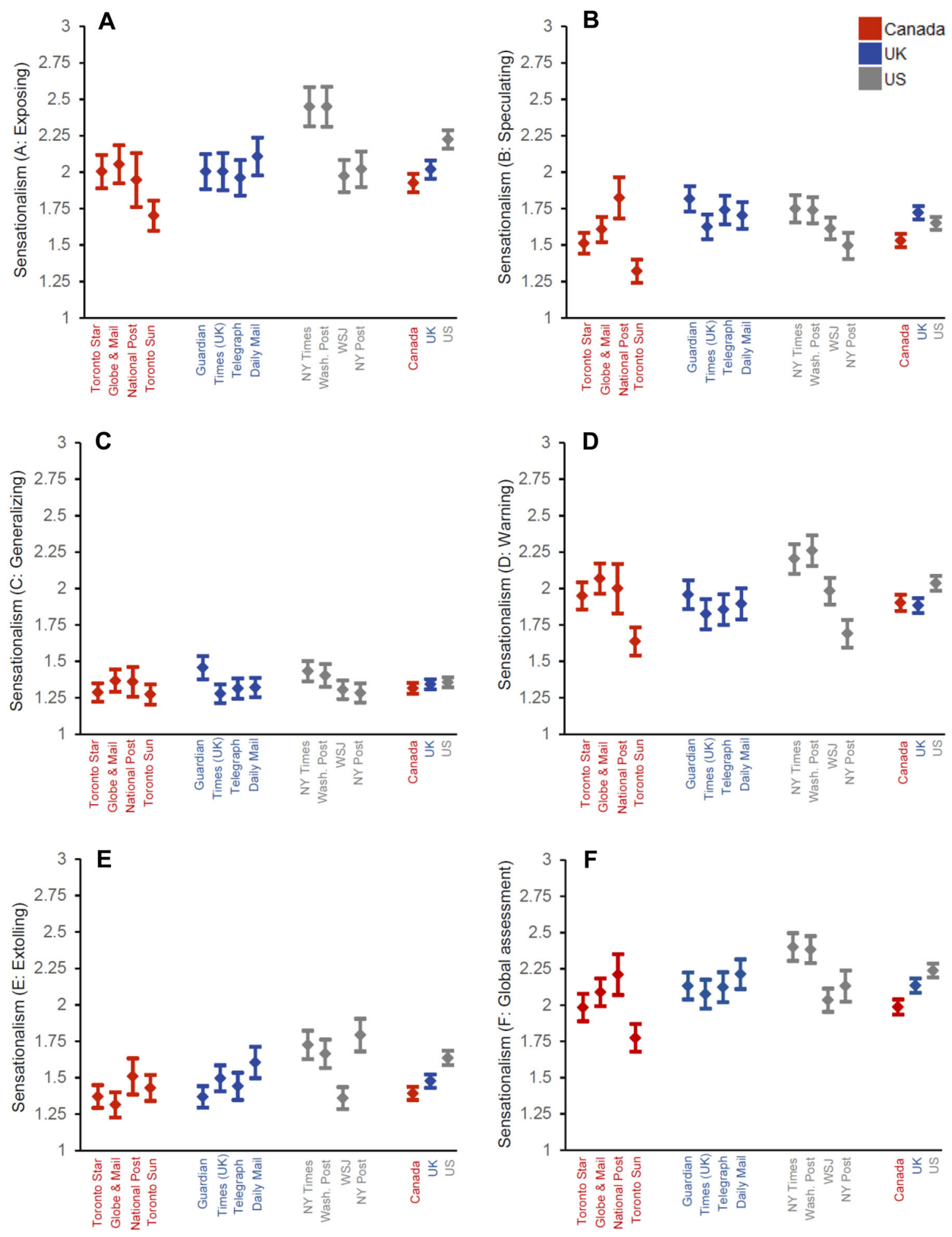

Fig. 4 Sensationalism of news media articles by news outlet and country of publication. Scores for six sensationalism questions (SI Coding Tool) are shown (mean, 95\% confidence interval) for articles ( $n=1331$ ) communicating COVID-19 public health or policy information (Fig. 2): (A) exposing, (B) speculating, (C) generalizing, (D) warning, (E) extolling, and (F) global assessment (i.e., an overall assessment of the degree of sensationalism in the article). Each question was evaluated on a scale from 1 to 5 ( 5 corresponding to highest sensationalism). Sampled articles were published between 1 March and 15 August 2020 (Methods). Kruskal-Wallis one-way analysis of variance and post-hoc multi-comparison test statistics are in SI Table S3.

on 3 July 2020 resulting from limited coverage of the healthcare sector that day (Fig. 5, SI Fig. S7).

The topics of pandemic media coverage. News media articles were categorized based on the societal sectors (up to 2 per article) that were the primary focus of each article (Fig. 5). The sectors, related to healthcare, leisure and entertainment, economics and commerce, government and politics, and other social services, are listed in full in Figs. 5 and 6. Although all analyzed articles contained information on the public-health effects of COVID-19 

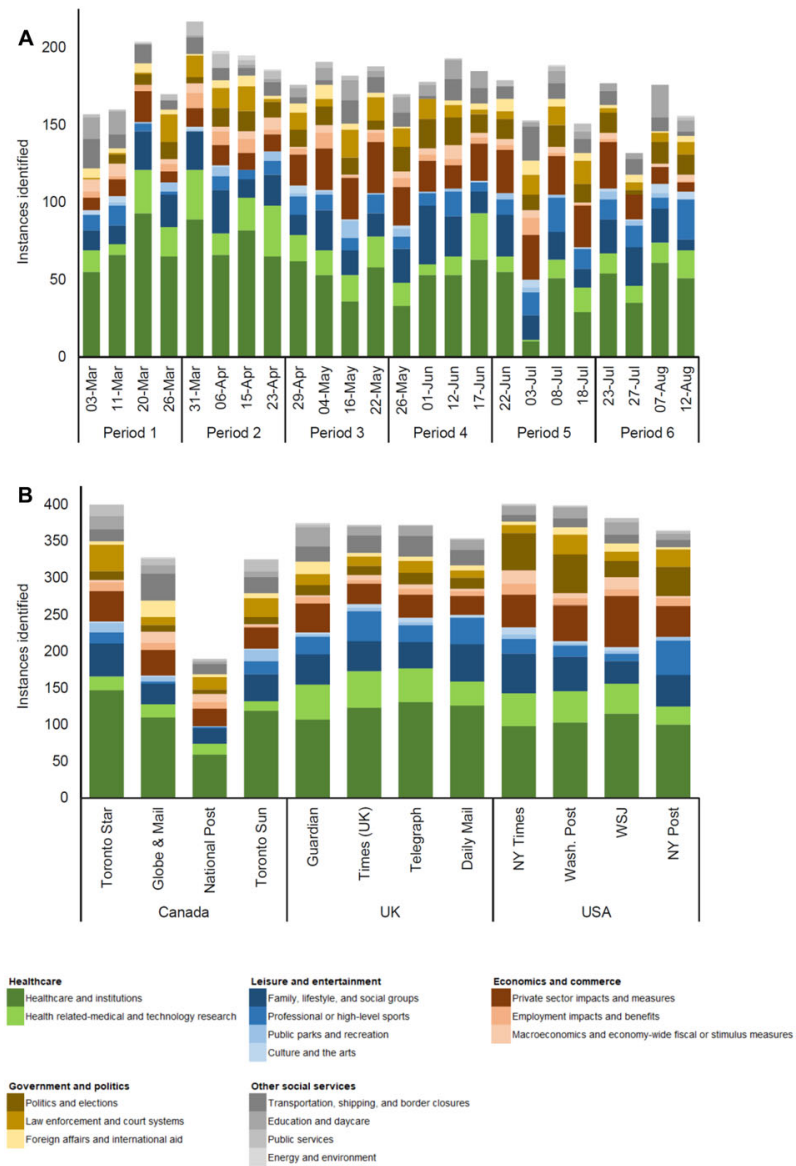

Fig. 5 COVID-19 news media article topics. The topics of news media articles analyzed (A) over the timeframe of this study and (B) by news outlet are specified. Sampled articles were published on randomly sampled days between 1 March and 15 August 2020 (Methods). The topic of each article $(n=1331)$ was categorized by societal sectors (up to 2 selected per article) related to healthcare, leisure and entertainment, economics and commerce, government and politics, and other social services.

or measures to limit its spread (SI Fig. S4), topics of focus differed widely, for example including recreation, the arts, transportation, or daycare, not just medical facilities or vaccine research.

The topics of news media articles corresponded to scientific quality and sensationalism of news reporting and analysis to some degree (Fig. 6). News media articles related to healthcare, health institutions, and health-related research were most common (Fig. 5), and they had significantly greater scientific quality compared to articles on other topics (mean global scientific quality of 3.23 for healthcare and institutions and 3.72 for health-related research; $p \leq 0.001$ for Kruskal-Wallis, $p \leq 0.05$ for pairwise comparisons except with energy and the environment; Fig. 6A). News media articles during the first four-week period studied, starting 1 March 2020, included the greatest focus (50.2\% of coverage) on healthcare and related institutions and research (Fig. 5A).

Sensationalism of articles related to politics and foreign affairs was greatest (mean global sensationalism of 2.53 for politics; and of 2.49 for foreign affairs; $p<0.001$ for Kruskal-Wallis, $p<0.05$ for pairwise comparisons of politics versus all sectors except foreign affairs, employment, and energy and the environment; Fig. 6B). For example, sensational statements related to politics and foreign affairs could include exposing disinformation from political leaders or extolling political leaders for border closures as a pandemic or broader policy response. News outlets in the
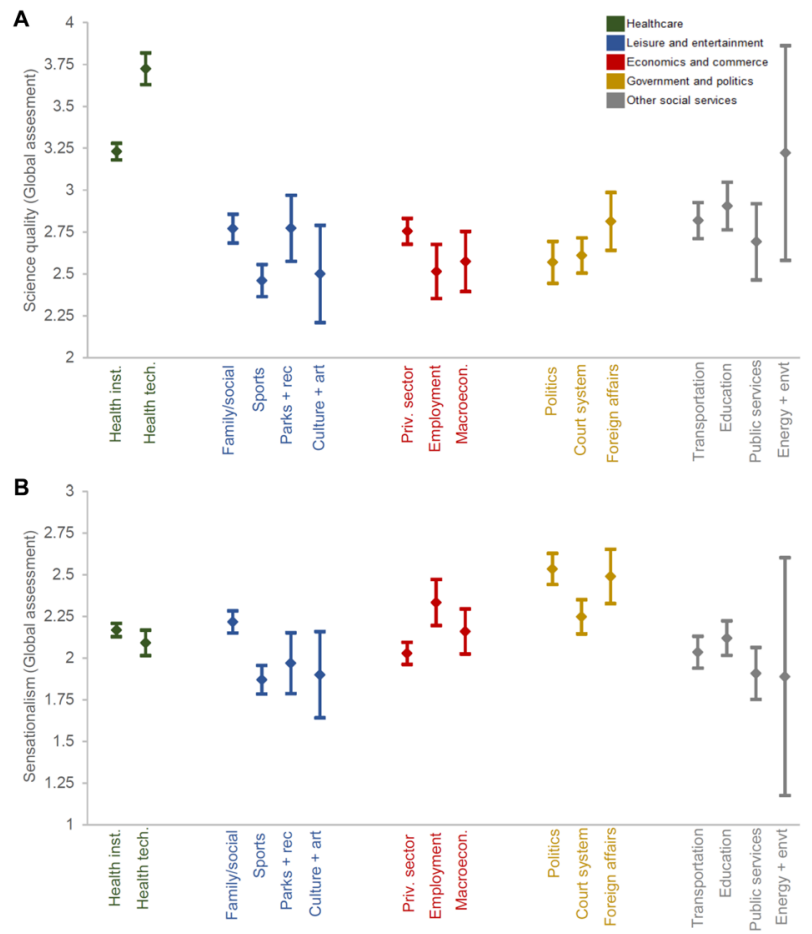

Fig. 6 Scientific quality and sensationalism across COVID-19 news media article topics. Scientific quality $(\mathbf{A})$ and sensationalism (B) of news media articles are indicated by the topics of articles. Overall global assessment scores for scientific quality and sensationalism (SI Coding Tool) are shown (mean, 95\% confidence interval) for articles communicating COVID-19 public health or policy information (Fig. 2). For each article, scientific quality and sensationalism were each evaluated on a scale from 1 to 5 ( 5 corresponding to highest scientific quality or to highest sensationalism). Sampled articles $(n=1331)$ were published between 1 March and 15 August 2020 (Methods). The topic of each article was categorized across the following societal sectors (up to two selected per article): healthcare and institutions; health-related medical and technology research; family, lifestyle, and social groups; professional or high-level sports; public parks and recreation; culture and the arts; private sector impacts and measures; employment impacts and benefits; macroeconomics and economy-wide fiscal or stimulus measures; politics and elections; law enforcement and court systems; foreign affairs and international aid; transportation, shipping, and border closures; education and daycare; public services; and energy and the environment.

United States published the most articles related to politics and elections (63.8\% of coverage across all outlets; Fig. 5B).

Public-health policies consistently covered through time included measures related to social distancing, testing and tracing, and protective equipment and disinfection practices, while coverage of mask guidance and reopening policies increased over the course of the study (Fig. 7).

\section{Discussion}

Managing the public health and societal risks of a pandemic requires iterative, informed decision-making by governments, individuals, and the private sector. News media play a central role in communicating public health and policy information, establishing accountability for decision-making, and shaping public perceptions through the number of news reports, their content, and their tone (Klemm et al., 2016; Reintjes et al., 2016). For news outlets spanning the political spectrum of three countries with contrasting public-health outcomes and policy responses (Fig. 1), based on a random sample of days, coverage related to COVID-19 

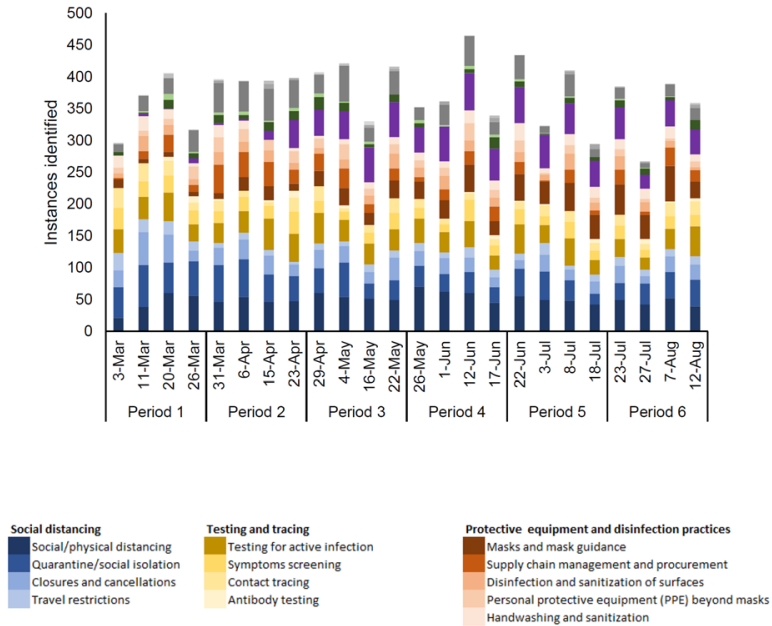

Reopening policies
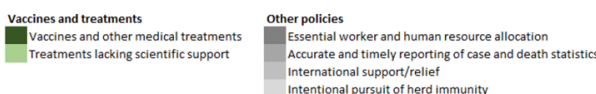

Fig. 7 The public-health policies and measures mentioned in news media articles analyzed over the timeframe of this study. Sampled articles ( $n=1331)$ were published on randomly sampled days between 1 March and 15 August 2020 (Methods). Public-health policies and measures in each article were coded under specific categories related to social distancing, testing and tracing, protective equipment and disinfection practices, reopening policies, vaccines and treatments, and more (all relevant categories selected for each article).

increased substantially in March 2020 and declined gradually thereafter in May and June (Fig. 2), not rebounding even during the dramatic increase in U.S. COVID-19 cases in June and July (SI Figure S5). Understanding this news media reporting in the early stages of COVID-19 response provides important lessons for ensuring the accessibility of information in support of public health and gauging its degree of effectiveness in creating accountability for policy decisions.

News media reporting grappled with complications of scientific understanding and its uncertainties during the timeframe of our study, as assessed through our measures of validity, precision, and overall scientific quality. For example, the mechanisms of disease transmission, especially airborne transmission, were slow to be recognized, leading to dynamic adjustments of public-health guidance (e.g., for mask usage by the general public) (Zhang et al., 2020). Despite such uncertainties and frequent knowledge updates over time, the scientific quality of reporting was highest for the healthcare sector, also the most commonly occurring article topic (Fig. 6). The scientific quality of reporting overall did not improve as the pandemic proceeded and knowledge of COVID-19 increased, which may be attributed to shifts from healthcare to other topics of news media reporting (Fig. 5 and SI Fig. S7).

We did, however, identify major differences in the degree to which newspaper reporting of COVID-19 presented high-quality scientific information about the public-health effects of the coronavirus disease and measures to limit its spread. News media articles generally had moderate scientific quality overall (Fig. 3F). Outlets on the populist-right of the political spectrum of each country, though, had significantly lower scientific quality in reporting related to COVID-19 (Fig. 3F). Scientific quality was low especially for validity, precision, and context as measures of scientific quality, as well as for the distinction between opinion versus facts in some cases (e.g., articles reporting claims without fact checking, specificity, background details, or sourcing) (Fig. 3). These findings pertain to news reporting and analysis, rather than opinion pieces, editorials, or letters, which were excluded from the scope of news media articles we evaluated. The differences across outlets suggest that, in reading news reporting and analysis in different newspapers, readers access reporting of varying scientific quality related to the health risks and effectiveness of available measures to limit disease transmission.

Further, patterns of U.S. media reporting were correlated with failures of national leadership under the Trump Administration, and they may have both reflected and contributed to politicization of COVID-19 in the United States. During this study's timeframe, the United States led the world in cases and deaths despite its pre-pandemic ranking as the country best equipped to manage a pandemic such as COVID-19 (Cameron et al., 2019). These public-health outcomes occurred against a backdrop of disinformation and failures of national leadership (Evanega et al., 2020; Ball and Maxmen, 2020; Holtz et al., 2020; Lincoln, 2020; Thorp, 2020). Lack of national leadership was observed in the relative dearth of national-level public-health policies and guidance (Fig. 1) and the divergence of subnational policy responses, correlated with partisan politics (SI Fig. S1 and Table S1). Elites and incumbent governments have outsize influence on public opinion and media coverage, which likely contributed to polarization and politicization of pandemic media coverage (Green et al., 2020; Hart et al., 2020). Linked to these trends, we observed higher sensationalism related to politics and elections topics and greater coverage of these sectors among U.S. newspapers (Figs. 5-6). Additionally, news outlets on the political left in the United States (i.e., New York Times, Washington Post) published articles with more exposing and warning coverage, for example discussing disinformation on the part of government leaders and the risks of disease (Fig. 4). Although most Americans believe the media are fulfilling key roles during the pandemic, the majority of these individuals identify as Democrats, and Democrats trust many more new sources than individuals identifying as Republican (Jurkowitz et al., 2020; Gottfried et al., 2020).

In both Canada and the United States, low scientific quality was paired with lower-than-average sensationalism in news outlets on the populist-right (Figs. 3F and $4 \mathrm{~F}$ ). Sensationalism was low overall for all news outlets, but within Canada and the United States, it was lowest for the Toronto Sun and New York Post, as well as the Wall Street Journal. Although low sensationalism is generally considered beneficial, very low sensationalism combined with low scientific quality may have failed to alert readers to public-health risks and policy failures in some cases (e.g., per the measures of exposing and warning coverage in Fig. 4). Such trends also resulted, in part, from higher reliance on syndicated articles, especially in Canada, potentially related to structural and economic changes in news media (SI Fig. S6). Across the political spectrum, our results demonstrate that existing ideological perspectives may influence how information is used in reporting (Rosella et al., 2013). For example, news outlets at the populistright in the United Kingdom and the United States may tend towards support of populist-right governments, demonstrating preference for those governments' interpretation of the science, implemented policies, and use of science to justify choices made (Bennett et al., 2008; Grundmann and Stehr, 2012).

The studied news media outlets-traditional, national-level print media-have disproportionate influence on the content of other media platforms and on how that content is covered (Project for Excellence in Journalism, 2010; Denham, 2014). A better understanding of the effects of news media-or lack thereof -on public-health decision-making and public sentiment in the early stages of this pandemic can, for future pandemics or other public-health crises, increase public-health officials' capacity to adapt communication strategies in disseminating guidance and coordinating responses of health system stakeholders (Laing, 
2011; Rosella et al., 2013; Klemm et al., 2016; Hoffman and Justicz, 2016; Pieri, 2019). Such understanding is crucial as the impacts of the policy actions themselves accumulate. The findings of this study point to complex interactions among scientific evidence on public-health risks and response measures, societal politicization of the science, and the scientific quality and sensationalism of media reporting. An inherent tension may exist: tendencies towards low sensationalism, especially combined with low scientific quality, may in some cases lead to characterization of public-health threats and policy failures as less extraordinary and relevant than they actually are.

\section{Data availability}

All data generated or analyzed during this study are included in this published article and its Supplementary Information.

Received: 6 January 2021; Accepted: 15 September 2021; Published online: 28 September 2021

\section{References}

Adeel AB, Catalano M, Catalano O, et al (2020) COVID-19 policy response and the rise of the sub-national governments. Can Public Policy. https://doi.org/ 10.3138/cpp.2020-101

Anderson B, Coletto D (2017) Canadian news media and "fake news" under a microscope. In: Abacus Data. https://abacusdata.ca/canadian-news-mediaand-fake-news-under-a-microscope/. Accessed 25 Aug 2020

Antoine J-Y, Villaneau J, Lefeuvre A (2014) Weighted Krippendorff's alpha is a more reliable metrics for multi- coders ordinal annotations: experimental studies on emotion, opinion and coreference annotation. In: Proceedings of the 14th conference of the European chapter of the Association for Computational Linguistics, Association for Computational Linguistics, Gotenborg, Sweden, pp. 550-559

Austen I (2020) Canada's largest newspaper changes hands amid vow to keep liberal voice. N. Y. Times

Ball P, Maxmen A (2020) The epic battle against coronavirus misinformation and conspiracy theories. Nature 581:371-374. https://doi.org/10.1038/d41586020-01452-z

Bennett WL, Lawrence RG, Livingston S (2008) When the press fails: political power and the news media from Iraq to Katrina. University of Chicago Press

Cameron EE, Nuzzo JB, Bell JA (2019) GHS index: global health security index: building collective action and accountability. NTI and Johns Hopkins Bloomberg School of Public Health

CBC News (2020) Tracking the spread of coronavirus in canada and around the world. https://newsinteractives.cbc.ca/coronavirustracker/. Accessed 25 Aug 2020

Centers for Disease Control and Prevention (CDC) (2020) CDC COVID Data Tracker. https://covid.cdc.gov/covid-data-tracker. Accessed 30 Aug 2020

Cohen J (1960) A coefficient of agreement for nominal scales. Educ Psychol Meas 20:37-46. https://doi.org/10.1177/001316446002000104

Denham BE (2014) Intermedia attribute agenda setting in the New York Times: the case of animal abuse in U.S. horse racing. Journal Mass Commun Q 91:17-37. https://doi.org/10.1177/1077699013514415

Evanega S, Lynas M, Adams J, Smolenyak K (2020) Coronavirus misinformation: quantifying sources and themes in the COVID-19 'infodemic.' Cornell Alliance for Science

Fleiss JL, Cohen J (1973) The equivalence of weighted kappa and the intraclass correlation coefficient as measures of reliability. Educ Psychol Meas 33:613-619. https://doi.org/10.1177/001316447303300309

Gentzkow M, Shapiro JM (2010) What drives media slant? Evidence from U.S. daily newspapers. Econometrica 78:35-71. https://doi.org/10.3982/ ECTA7195

Giraudoux P, Antonietti J-P, Beale C, et al (2018) pgirmess: spatial analysis and data mining for field ecologists. R package version 1.6.9 https://CRAN.Rproject.org/package $=$ pgirmess

Gottfried J, Walker M, Mitchell A (2020) Americans' views of the news media during the Coronavirus outbreak. Pew Research Center

Government of Canada (2016) Canada's health care system. https:// www.canada.ca/en/health-canada/services/canada-health-care-system.html. Accessed 18 Oct 2020

Green J, Edgerton J, Naftel D et al. (2020) Elusive consensus: polarization in elite communication on the COVID-19 pandemic. Sci Adv 6:eabc2717. https:// doi.org/10.1126/sciadv.abc2717
Grossman G, Kim S, Rexer JM, Thirumurthy H (2020) Political partisanship influences behavioral responses to governors' recommendations for COVID19 prevention in the United States. Proc Natl Acad Sci USA 117:24144-24153. https://doi.org/10.1073/pnas.2007835117

Grundmann R, Stehr N (2012) The power of scientific knowledge: from research to public policy. Cambridge University Press, Cambridge

Hallgren KA (2012) Computing inter-rater reliability for observational data: an overview and tutorial. Tutor Quant Methods Psychol 8:23-34. https:// doi.org/10.20982/tqmp.08.1.p023

Hart PS, Chinn S, Soroka S (2020) Politicization and polarization in COVID-19 news coverage. Sci Commun 42:679-697. https://doi.org/10.1177/1075547020950735

Hoffman SJ, Justicz V (2016) Automatically quantifying the scientific quality and sensationalism of news records mentioning pandemics: validating a maximum entropy machine-learning model. J Clin Epidemiol 75:47-55. https:// doi.org/10.1016/j.jclinepi.2015.12.010

Holtz D, Zhao M, Benzell SG et al. (2020) Interdependence and the cost of uncoordinated responses to COVID-19. Proc Natl Acad Sci USA 117:19837-19843. https://doi.org/10.1073/pnas.2009522117

Hönnige C, Nyhuis D, Meyer P et al. (2020) Dominating the debate: visibility bias and mentions of British MPs in newspaper reporting on Brexit. Polit Res Exch 2:1788955. https://doi.org/10.1080/2474736X.2020.1788955

Jurkowitz M, Mitchell A, Shearer E, Walker M (2020) U.S. media polarization and the 2020 election: a nation divided. Pew Research Center

Klemm C, Das E, Hartmann T (2016) Swine flu and hype: a systematic review of media dramatization of the H1N1 influenza pandemic. J Risk Res 19:1-20. https://doi.org/10.1080/13669877.2014.923029

Lacy S, Riffe D, Stoddard S et al. (2001) Sample size for newspaper content analysis in multi-year studies. J Mass Commun Q 78:836-45

Laing A (2011) The H1N1 crisis: roles played by government communicators, the public and the media. J Prof Commun 1:123-149. https://doi.org/10.15173/ jpc.vli1.88

Lincoln M (2020) Study the role of hubris in nations' COVID-19 response. Nature 585:325-325. https://doi.org/10.1038/d41586-020-02596-8

Mitchell A, Simmons K, Matsa KE, et al (2018) In Western Europe, public attitudes toward news media more divided by populist views than left-right ideology. Pew Research Center

Molek-Kozakowska K (2013) Towards a pragma-linguistic framework for the study of sensationalism in news headlines. Discourse Commun 7:173-197. https:// doi.org/10.1177/1750481312471668

Oxman AD, Guyatt GH, Cook DJ et al. (1993) An index of scientific quality for health reports in the lay press. J Clin Epidemiol 46:987-1001. https://doi.org/ 10.1016/0895-4356(93)90166-x

Pieri E (2019) Media framing and the threat of global pandemics: the Ebola crisis in UK media and policy response. Sociol Res Online 24:73-92

Project for Excellence in Journalism (2010) How news happens: a study of the news ecosystem of one American city. Pew Research Center

Public Health England, NHSX (2020) Coronavirus (COVID-19) in the UK. https:// coronavirus.data.gov.uk. Accessed 2 Oct 2020

Puglisi R, Snyder JM (2015) The balanced US press. J Eur Econ Assoc 13:240-264. https://doi.org/10.1111/jeea.12101

R Core Team (2020) R: a language and environment for statistical computing. $R$ Foundation for Statistical Computing, Vienna, Austria

Reintjes R, Das E, Klemm C et al. (2016) "Pandemic public health paradox": time series analysis of the 2009/10 influenza A / H1N1 epidemiology, media attention, risk perception and public reactions in 5 European countries. PLoS ONE 11:e0151258. https://doi.org/10.1371/journal.pone.0151258

Riffe D, Aust CF, Lacy SR (2016) The effectiveness of random, consecutive day and constructed week sampling in newspaper content analysis. Journal Q 70:133-139. https://doi.org/10.1177/107769909307000115

Rosella LC, Wilson K, Crowcroft NS et al. (2013) Pandemic H1N1 in Canada and the use of evidence in developing public health policies-A policy analysis. Soc Sci Med 83:1-9. https://doi.org/10.1016/j.socscimed.2013.02.009

Roser M, Ritchie H, Ortiz-Ospina E, Hasell J (2020) Coronavirus pandemic (COVID-19). Our World in Data. https://ourworldindata.org/coronavirus. Accessed 30 Aug 2020

Thorp HH (2020) Trump lied about science. Science 369:1409. https://doi.org/ $10.1126 /$ science.abe7391

Tran D, Dolgun A, Demirhan H (2020) Weighted inter-rater agreement measures for ordinal outcomes. Commun Stat-Simul Comput 49:989-1003. https:// doi.org/10.1080/03610918.2018.1490428

World Health Organization (WHO) (2020a) Coronavirus disease (COVID-19) pandemic. https://www.who.int/emergencies/diseases/novel-coronavirus2019. Accessed 7 Jun 2020

World Health Organization (WHO) (2020b) Timeline of WHO's response to COVID-19. https://www.who.int/news/item/29-06-2020-covidtimeline. Accessed 5 Jun 2020

Zhang R, Li Y, Zhang AL et al. (2020) Identifying airborne transmission as the dominant route for the spread of COVID-19. Proc Natl Acad Sci USA 117:14857-14863. https://doi.org/10.1073/pnas.2009637117 


\section{Acknowledgements}

S. Damouras provided advising on methods of statistical analysis, and J. Niemann formatted references. Funding for this work was provided by the University of Toronto Scarborough Department of Physical and Environmental Sciences and the University of Miami Rosenstiel School of Marine and Atmospheric Science.

\section{Author contributions}

All authors conceived the analysis. KJM, RSR, BP, JT, CAC, SGC, KET, and NK designed the methods of analysis with review by all authors. RSR, BP, JT, CAC, SGC, and KET collected data. KJM, RSR, BP, and JT performed analysis of data and developed visualizations of data. KJM, RSR, BP, JT, and NK drafted the manuscript with review and edits from all authors.

\section{Competing interests}

The authors declare no competing interests.

\section{Additional information}

Supplementary information The online version contains supplementary material available at https://doi.org/10.1057/s41599-021-00900-z.
Reprints and permission information is available at http://www.nature.com/reprints

Publisher's note Springer Nature remains neutral with regard to jurisdictional claims in published maps and institutional affiliations.

(c) (i) Open Access This article is licensed under a Creative Commons Attribution 4.0 International License, which permits use, sharing, adaptation, distribution and reproduction in any medium or format, as long as you give appropriate credit to the original author(s) and the source, provide a link to the Creative Commons license, and indicate if changes were made. The images or other third party material in this article are included in the article's Creative Commons license, unless indicated otherwise in a credit line to the material. If material is not included in the article's Creative Commons license and your intended use is not permitted by statutory regulation or exceeds the permitted use, you will need to obtain permission directly from the copyright holder. To view a copy of this license, visit http://creativecommons.org/ licenses/by/4.0/.

(C) The Author(s) 2021

Correspondence and requests for materials should be addressed to Katharine J. Mach. 